\title{
A quick glance at selected topics in this issue
}

\author{
Pradeep Bhambhvani, MD, ${ }^{\mathrm{a}}$ Fadi G. Hage, MD, MASNC, ${ }^{\mathrm{b}, \mathrm{c}}$ and \\ Ami E. Iskandrian, MD, MASNC ${ }^{\mathrm{b}}$ \\ a Division of Molecular Imaging and Therapeutics, Department of Radiology, The University of \\ Alabama at Birmingham, Birmingham, AL \\ b Division of Cardiovascular Disease, The University of Alabama at Birmingham, Birmingham, AL \\ c Section of Cardiology, Birmingham Veterans Affairs Medical Center, Birmingham, AL
}

Received Sep 10, 2020; accepted Sep 11, 2020

doi: $10.1007 / \mathrm{s} 12350-020-02384-\mathrm{x}$

"A quick glance at selected topics in this issue" aims to highlight contents of the Journal and provide a quick review to the readers.

Key Words: CAD • Diseases/processes • Amyloid heart disease $\bullet$ PET $・$ Modalities $・$ SPECT $•$ Hybrid imaging $\cdot$ MPI $\cdot$ Tests

\begin{tabular}{|c|c|c|c|}
\hline & MBF & Myocardial blood flow \\
\hline \multicolumn{2}{|c|}{$\begin{array}{l}\text { Abbreviations } \\
\text { CAD Coronary artery disease }\end{array}$} & MFR & Myocardial flow reserve \\
\hline \multirow{3}{*}{$\begin{array}{l}\text { MPI } \\
\text { SPECT }\end{array}$} & Myocardial perfusion imaging & DPD & Diphosphono-1,2-propanodicarboxylic \\
\hline & Single photon emission computed & & \\
\hline & tomography & ATTR & Transthyretin cardiac amyloidosis \\
\hline PET & Positron emission tomography & MACE & Major adverse cardiac events \\
\hline LV & Left ventricle & & \\
\hline LVEF & Left ventricle ejection fraction & & \\
\hline
\end{tabular}

"A quick glance at selected topics in this issue" aims to highlight contents of the Journal and provide a quick review to the readers. Every issue of the journal has multiple entries with differing objectives. We realize that many of you do not have time to read all journals or attend all national meetings. For that reason, JNC includes 2 types of literature reviews. One summarizing recent key nuclear cardiology articles that have been published in journals other than ours (https://doi.org/10. 1007/s12350-020-02347-2) while the second outlines select publications in the general cardiovascular disease literature that have relevance to our field (https://doi.org/

Reprint requests: Pradeep Bhambhvani, MD, Division of Molecular Imaging and Therapeutics, Department of Radiology, The University of Alabama at Birmingham, 619 19th Street South JT 777, Birmingham, AL 35249; pbhambhvani@uabmc.edu

J Nucl Cardiol 2020;27:1425-8.

$1071-3581 / \$ 34.00$

Copyright (C) 2020 American Society of Nuclear Cardiology. 10.1007/s12350-020-02364-1). Another entry is the historical corner that in the current issue looks at the career and scientific contributions of an eminent physician and ASNC founder Frans J Th Wackers, MD, PhD (Born 1939) (https://doi.org/10.1007/s12350-020-02351 -6). These manuscripts are complimented by a great selection of original articles with accompanying editorials, brief reports, 'What is this Image' and 'Images that Teach' and a CME review paper, which currently is on 'Myocardial Perfusion Imaging for Diabetes Mellitus' by Acampa and colleagues from University Federico II, Naples, Italy (https://doi.org/10.1007/s12350-019-0184 6-1). Many of the original articles also have accompanying PowerPoint slides. The abstract of the lead original article 'Effect of proton pump inhibitors on Rubidium-82 gastric uptake using PET MPI' by Alzahrani et al. from University of Ottawa, Canada, has also been translated into Spanish, Chinese and French in keeping with the growing international readership. PowerPoint slides from this paper can be found by 
searching (https://doi.org/10.1007/s12350-019-01954-y ). Also included is an information statement from ASNC, IAEA, and SNMMI on 'Guidance and best practices for reestablishment of non-emergent care in nuclear cardiology laboratories during the coronavirus disease (COVID-19) pandemic (https://doi.org/10.1007/ s12350-020-02203-3).

Our comments on a few selected papers noted below are therefore only the tip of the iceberg. These manuscripts were selected at random and we sincerely believe all original articles serve a purpose, provide great value and have undergone an intense peer review.

$\mathrm{Rb}-82$ PET MPI is a well validated tool for CAD evaluation with very good sensitivity and specificity. However, prominent gastric uptake with Rb-82 PET MPI is seen in $10 \%$ of studies and commonly results in inferior myocardial attenuation and thus may affect image quality and diagnostic accuracy of myocardial ischemia. Alzahrani and colleagues from University of Ottawa, Canada (https://doi.org/10.1007/s12350-019-01 954-y), attempt to understand the relationship between $\mathrm{Rb}-82$ gastric uptake and proton pump inhibitors (PPI) use. Of the 600 enrolled patients for a clinically indicated Rb-82 PET MPI, 181 (30.2\%) patients were using PPI. The Rb-82 gastric uptake appeared to be greater in patients actively using PPI, a potential set up for nondiagnostic PET MPI. The authors find that gastric uptake was lower if patients had stopped PPIs for $\geq 36$ hours.

Singh et al. from PGIMER, Chandigarh, India (h ttps://doi.org/10.1007/s12350-019-01733-9), study the association of gastric wall uptake with PPI intake on stress/rest SPECT MPI and compare its incidence with $\mathrm{H} 2$ antagonists (H2A) users and a control group of patients not on either gastroprotective medication $(n=156)$. Clinically relevant gastric wall uptake was seen in $36 \%$ of PPI group patients compared to $8 \%$ in the control group, $10.5 \%$ in the H2A group, and $9.5 \%$ in the intervention group (PPI discontinued for 3 days before MPI) respectively, with statistically significant difference. PPI therapy, irrespective of duration was associated with higher incidence of clinically relevant gastric wall uptake which may affect the diagnostic accuracy of MPI. Patients scheduled for vasodilator stress MPI may be advised to stop PPIs for a minimum of 3 days prior or replace them with $\mathrm{H} 2 \mathrm{~A}$ in presence of significant dyspeptic symptoms.

Bone scintigraphy with 99mTc-DPD and other similar tracers is a proven technique for accurate diagnosis of transthyretin cardiac amyloidosis (ATTR). Caobelli et al. from University of Basel, Switzerland (h ttps://doi.org/10.1007/s12350-019-01893-8) demonstrate the feasibility of quantitative 99mTc-DPD scintigraphy in 13 patients suspected with ATTR cardiac amyloidosis using various parameters and then correlate them to the validated Perugini visual score. Myocardial SUVmax and SUVpeak and respective bone normalized values showed a fairly strong correlation with Perugini score. There was a great overlap of quantitative values in patients with Perugini score 2 and 3 . Thus quantitation may allow assessment of cardiac amyloid burden, with potential for prognostic stratification, evaluation of disease progression and treatment monitoring.

Sperry and colleagues (https://doi.org/10.1007/s12 350-020-02139-8) analyze 109 consecutive patients who underwent technetium pyrophosphate nuclear scintigraphy for the evaluation of transthyretin cardiac amyloidosis. Patients were imaged at 1 and 3 hours after radiotracer injection using both planar and SPECT/CT, and correlations between imaging protocols were compared. Findings consistent with ATTR amyloidosis on planar images included a semiquantitative grade of 2-3 and $\mathrm{H} / \mathrm{CL}$ ratio $\geq 1.5$ on 1 -hour images and $\geq 1.3$ on 3 hour images. SPECT and CT images were graded qualitatively as positive (focal or diffuse) or negative for left ventricular myocardial uptake. The study has two important messages. First, it showed that planar imaging alone is not sufficient for diagnosis of ATTR cardiac amyloidosis. There was significant discordance in studies when comparing planar imaging to SPECT/CT at both time points (16\% of the cohort were considered indeterminate or misclassified). Second, the imaging time point ( 1 hour vs. 3 hour) did not significantly affect the interpretation of the study.

Patients who achieve $\geq 10$ METS during exercise SPECT MPI have very low rates of significant ischemia and major adverse cardiac events (MACE). Smith and colleagues from University of Virginia Health System, Charlottesville, Virginia, USA (https://doi.org/10.1007/ s12350-018-1376-7) examined the workload achieved, prevalence and predictors of ischemia, and MACE (cardiac death, non-fatal MI, late revascularization) in a cohort of 382 patients' $\geq 65$ years of age who underwent exercise 99m-Tc SPECT MPI. A substantial proportion of older adults $(\sim 25 \%)$ were able to achieve $\geq 85 \%$ maximum age-predicted heart rate and $\geq 10$ METS. This subgroup had low rates of significant LV ischemia $[3.1 \%$ prevalence of $\geq 10 \% \mathrm{LV}$ ischemia (1.2\% in those without ST depression)]. Cardiac death and MACE rates were low at $0.6 \%$ /year and $2.6 \%$ year over a median 7 years of follow-up. In these low-risk patients a provisional imaging protocol could be established, where the imaging agent need not be injected at peak exercise unless other adverse exercise test endpoints are present (e.g., typical anginal chest pain, exercise hypotension, serious arrhythmias etc.).

Patients with atrial fibrillation (AF) are known to have increased FDG uptake in the atria. Xie et al. from Capital Medical University, Beijing, China (https://doi. 
org/10.1007/s12350-018-1387-4) explore the factors that are associated with increased atrial FDG uptake in 48 AF patients. Atrial uptake was present in patients with persistent $\mathrm{AF}$ and localized mainly in the right atrium, whereas bilateral atrial appendage uptake could be equally involved. Multiple factors contributed to the increased atrial activity; in particular, the epicardial adipose tissue activity was independently correlated with the activity of the atrium and the atrial appendage.

Doukky and colleagues from Chicago (https://doi. org/10.1007/s12350-018-1415-4), examine the prognostic significance of ischemic electrocardiographic changes during regadenoson stress MPI. Among 8615 subjects, $89(1.0 \%)$ had ST depression $\geq 1.0 \mathrm{~mm}$ and 133 (1.5\%) had ST depression $\geq 0.5 \mathrm{~mm}$. Regadenoson-induced ischemic ST depression is more common in women and $\mathrm{ST}$ depression $\geq 0.5 \mathrm{~mm}$ provided a modest independent prognostic value (higher MACE: cardiac death, myocardial infarction, or coronary revascularization) beyond MPI and clinical parameters. The authors suggest that ST depression $\geq 0.5 \mathrm{~mm}$ is a better threshold than $\geq 1.0 \mathrm{~mm}$ to define ECG evidence for regadenoson-induced myocardial ischemia.

Young Seok et al. from the Samsung Medical Center, Seoul, Korea (https://doi.org/10.1007/s12350-0 18-1424-3) study the association of serum uric acid levels with carotid FDG uptake as a marker of inflammatory atherosclerosis in a cohort of 970 asymptomatic adults with no apparent cardiovascular disease. Higher serum uric acid quartiles were associated with greater triglyceride, and lower high-density lipoprotein cholesterol and estimated GFR levels. Additionally, elevated serum uric acid in these asymptomatic adults was associated with increased carotid FDG uptake, suggesting a potential role of hyperuricemia in carotid inflammatory atherosclerosis. Incidentally there was no correlation between serum uric acid level and carotid intima media thickness.

FDG PET is a validated imaging biomarker for assessment of vessel wall inflammation. Toczek and colleagues from Yale University School of Medicine, New Haven, CT (https://doi.org/10.1007/s12350-020-0 2055-x) study aortic wall FDG signal quantification in 13 subjects without prior history of cardiovascular disease who had enrolled in a study of vascular inflammation. Kinetic modeling supported the use of mean target to blood ratio (TBRmax, i.e., ratio of aortic mean SUVmax to blood pool activity measured in the superior vena cava and expressed as mean SUV) as a surrogate for net uptake rate of FDG in the arterial wall. Mean TBRmax was superior to mean SUVmax.

Dilated cardiomyopathy (DCM) is associated with marked morbidity, mortality and frequent hospitalizations. Presence of left ventricular systolic dyssynchrony
(LVSD) has been an important prognostic factor in the DCM patients. However, the association between the LV diastolic dyssynchrony (LVDD) and clinical outcome in DCM is not well established. Wang et al. (h ttps://doi.org/10.1007/s12350-018-01468-z) evaluate the prognostic value of both systolic and diastolic dyssynchrony in 52 patients with DCM using resting SPECT MPI. Compared with survivors, patients with cardiac death had lower LVEF and more severe LV systolic and diastolic dyssynchrony. Thus both the LVSD and LVDD parameters have important prognostic values for DCM patients. Both systolic and diastolic phase entropy were independent prognostic factors for cardiac death.

Fudim and colleagues (https://doi.org/10.1007/s12 350-019-01843-4) investigate the prevalence and prognostic value of diastolic and systolic dyssynchrony in a cohort of patients with CAD \pm heart failure (HF). Patients with $\mathrm{CAD}+\mathrm{HF}$ have a higher prevalence (30\%, twice as common) of mechanical dyssynchrony as measured by gated SPECT MPI, and a higher mortality than CAD alone. However, clinical outcomes associated with mechanical dyssynchrony did not differ in patients with and without HF.

Malik et al. from PGIMER, Chandigarh, India (h ttps://doi.org/10.1007/s12350-018-1436-z) examine gated SPECT MPI derived left ventricular mechanical dyssynchrony (LVMD) indices as predictors of major adverse cardiac events (MACEs) in long-standing diabetics with normal perfusion and systolic function. Patients experiencing MACEs showed significantly higher LVMD indices, i.e., phase standard deviation and wider phase bandwidth than those who did not. Additionally there was significant association between diabetes duration, microvascular complications, and LVMD indices for predicting MACE.

The significance of post-stress reduction in left ventricular ejection fraction (LVEF) in patients with normal perfusion on adenosine stress/rest SPECT MPI remains controversial. Obeidat and colleagues (https://d oi.org/10.1007/s12350-019-01725-9), retrospectively evaluate the frequency and prognostic value of postadenosine stress reduction in LVEF ( $\geq 5 \%$ ) in a cohort of 560 patients with normal adenosine stress/rest SPECT MPI. ECG changes suggestive of ischemia were seen in $11 \%$ of the patients. $\geq 5 \%$ reduction in $\mathrm{LVEF}$ was common (seen in $24.1 \%$ of patients) and both $\geq 5 \%$ or $\geq 10 \%$ reductions in LVEF did not confer adverse prognosis compared with patients without such reductions.

Garcia et al. (https://doi.org/10.1007/s12350-018-1 432-3) describe and validate an artificial intelligence (AI)-driven structured reporting system by direct comparison of automatically generated reports by an AI system to results from actual clinical reports of rest- 
stress SPECT MPI generated by one of nine nuclear cardiology experts. The AI reporting system had a diagnostic performance comparable to those of experts.

Non-alcoholic fatty liver disease (NAFLD) is an independent risk factor for cardiovascular disease. Tang and colleagues from the First Affiliated Hospital of Wenzhou Medical University, Zhejiang, China (https://d oi.org/10.1007/s12350-018-1446-x) investigate the association between NAFLD and myocardial glucose uptake assessed by 18F-FDG PET. Myocardial glucose uptake was significantly lower in individuals with NAFLD compared with those without fatty liver. Furthermore, NAFLD patients with lower myocardial glucose uptake were more likely to have higher proportion of increased LV filling pressure than that of patients with higher myocardial glucose uptake. This suggests that otherwise healthy subjects with NAFLD may be at risk of impaired myocardial glucose metabolism and diastolic heart dysfunction.

Doxorubicin is the most frequently used anthracycline and a cornerstone in lymphoma treatment. However, doxorubicin treatment is limited by dose dependent chronic cardiotoxicity that is typically defined as a decline in LVEF of more than 10 percentage points below the lower limit of normal. Laursen et al. from University of Copenhagen, Denmark (https://doi. org/10.1007/s12350-018-1458-6) investigate the value of rest-stress $82 \mathrm{Rb}$ PET MPI before and after the 1st doxorubicin chemotherapy for assessment of treatment effects on the myocardial microcirculation in patients with lymphoma. Notably myocardial perfusion reserve decreased significantly after the initial doxorubicin exposure (2.69 vs $2.51, \mathrm{P}=.03)$ and may represent an early sign of cardiotoxicity. A non-significant decline in stress perfusion was noted while there was no change in resting myocardial perfusion. Thus $82 \mathrm{Rb}$ PET derived myocardial perfusion reserve may serve as an early marker of doxorubicin induced cardiotoxicity.

Harel and colleagues from Montreal Heart Institute, Canada (https://doi.org/10.1007/s12350-018-01478-x) compare the effects of different dipyridamole infusion times on PET MPI derived stress MBF in 147 subjects with low likelihood of significant CAD. The 4-minute and 5-minute dipyridamole infusion protocols produce comparable myocardial flow response, hemodynamic changes, and symptoms, in subjects with low likelihood of significant obstructive CAD.

We encourage the readers to look at the several other articles in the Journal with accompanying scholarly and informative editorials that not only put the findings in perspectives but also outline future directions. We would like to hear your comments as we strive to gain knowledge and in the process, improve patient care.

\section{Disclosures}

The authors declare that they do not have conflict of interest.

Publisher's Note Springer Nature remains neutral with regard to jurisdictional claims in published maps and institutional affiliations. 